\title{
Markov mode-multiplexing mode in OFDM outphasing transmitters
}

\author{
Pedro L. Carro* (D), Paloma Garcia-Ducar, Jesus de Mingo and Antonio Valdovinos
}

\begin{abstract}
Outphasing transmitters have been explored to study the trade-off between linearity and efficiency. The outphasing technique enhances efficiency by operating two amplifiers at lower output amplitudes, using two constant envelope signals. Their major drawback is the inherent sensitivity to gain and phase imbalances between the two amplifier branches. Another important issue is related to the degradation of efficiency, especially in isolated combiners. This paper presents a Statistical Markov-Chain Mode-Multiplexing (MM) transmitter which combines features of the MM and Reverse MM-LINC. Commercial analog devices and a digital platform for signal processing purposes are used to test the performance with an orthogonal frequency multiplexing modulation (OFDM), which is one of the most used modulation schemes in wireless communication systems.
\end{abstract}

Keywords: PAPR, OFDM, Outphasing

\section{Introduction}

Radio frequency power amplifiers (PAs) are the most critical components in the design of spectral and power efficient wireless transmitters. Many transmitted signals in the new standards, such as long-term evolution (LTE), and the future $5 \mathrm{G}$ multicarrier-based modulation schemes such as Filtered Band Multicarrier (FBMC) and Generalized Frequency Division Multiplexing (GFDM) have a high peak-to-average power ratio (PAPR) caused by complex modulation schemes. The use of high PAPR signals requires a large enough back-off in the power amplifier operating to satisfy the stringent linearity requirement, but this region shows a very low PA efficiency. Recent trends in efficient and linear PA research are mainly focusing on the use of two-branch amplifier systems and moving away from the classical single-ended amplifier topology combined with the use of digital predistortion techniques. Among these dual-branch systems, the most popular are the Doherty amplifier, the envelope elimination and restoration (EER) techniques, the linear amplification with nonlinear components (LINC), and the modified implementation of the LINC (MILC technique) [1-3].

\footnotetext{
*Correspondence: plcarro@unizar.es

Department of Electronic Engineering and Communications, Aragon Institute of Engineering Research (I3A), University of Zaragoza, status=Mariano Esquillor, , 50018 Zaragoza, Spain
}

This paper presents a complete design and experimental implementation of a new Mode-Multiplexing LINC technique $[4,5]$ in order to enhance the efficiency with a reduced spectral regrowth. The paper is outlined as follows. In Section 2, we introduce the previous mode multiplexing methods applied in the context of the LINC. The novel Markov mode multiplexing method is explained in Section 3. The simulations, which have been accomplished to analyze the proposed technique, are shown in Section 4 . The proposed algorithm is validated by means of an experimental setup and the main results are discussed in Section 5. Finally, some conclusions about this work are provided in Section 6.

\section{Mode-multiplexed LINC methods}

There are some issues which decrease the overall performance in a LINC transmitter implementation, namely the power gain and phase imbalance between the two RF paths. These are typically due to PAs, mixers, path length differences, quadrature modulators errors, quantization noise, and sampling rate error [6-9]. A LINC scheme which is implemented with an isolated combiner shows an efficiency which is the product of those corresponding to both PA and combiner [2, 10, 11]. Although PA efficiency is maximized, the whole performance is dramatically reduced in a LINC structure if the combiner 
efficiency is taken into account. Therefore, some techniques, like the Mode-Multiplexing method (MM-LINC), have been proposed either to improve combiner efficiency or linearity (Reverse Mode, RM-LINC) [4].

Figure 1 shows a schematic diagram of the ModeMultiplexing LINC structure (MM-LINC). The source signal $s(n)$ (we use $s(n)=\left.s(t)\right|_{t=n \cdot T_{m}}$, being $T_{m}$ the sampling period to describe its discrete version) is split into two constant envelope signals of a LINC transmitter by a Signal Component Separator block (SCS).

The MM-LINC and the RM-LINC technique are applied at the signal component separator stage. In a classical implementation, the constant envelope signals are computed by means of

$$
\left\{\begin{array}{l}
s_{1}(n) \\
s_{2}(n)
\end{array}\right\}=\left[\begin{array}{cc}
1 & j e(n) \\
1 & -j e(n)
\end{array}\right]\left\{\begin{array}{l}
s(n) / 2 \\
s(n) / 2
\end{array}\right\}
$$

where $j e(n)$ is a signal which is in quadrature to the source signal, $s(n)=c(n) e^{j \rho(n)}$, and it is evaluated as

$$
e(n)=\sqrt{\frac{c_{\max }^{2}}{|s(n)|^{2}}-1} \quad 0<c(n)<c_{\max }
$$

where $c_{\text {max }}$ is the maximum of the signal envelope $c(n)$. If the LINC decomposition is expressed in matrix form,

$$
\mathbf{s}_{\mathrm{LINC}}(n)=\Theta(n) \mathbf{s}(n)
$$

the MM-LINC and the RM-LINC are easily introduced substituting the matrix $\Theta$ by

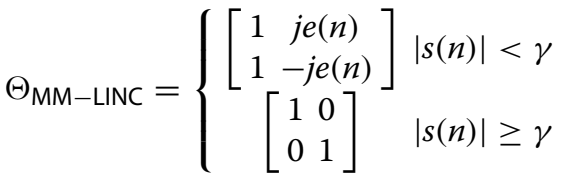

The MM-LINC switches between the so-called outphasing (constant envelope, here denoted by "O") and balanced modes (original envelope with $3-\mathrm{dB}$ backoff, denoted by "B"), shown in Fig. 1 as a state diagram. The MMLINC decomposition improves the LINC transmitter efficiency because the outphasing angle is reduced compared to the standard LINC solution. The reverse mode is obtained by inverting the inequalities in (4), achieving a better linearization instead of an efficiency improvement. The threshold $\gamma$ must be optimized for every desired transmitted waveform, and thus, the method performance depends on the signal probability density function. Authors propose a novel switching method (Markov mode-multiplexing) which allows to transmit any particular envelope value in certain time step as outphasing mode but as a balanced mode later.

\section{Markov chain MM-LINC technique}

\subsection{Algorithm principles}

The MM-LINC structure is modified through the signal component separation block, which is proposed to be

$\mathbf{s}_{\text {MARKOV-MM-LINC }}(n)=\Lambda(n) \mathbf{s}(n)$

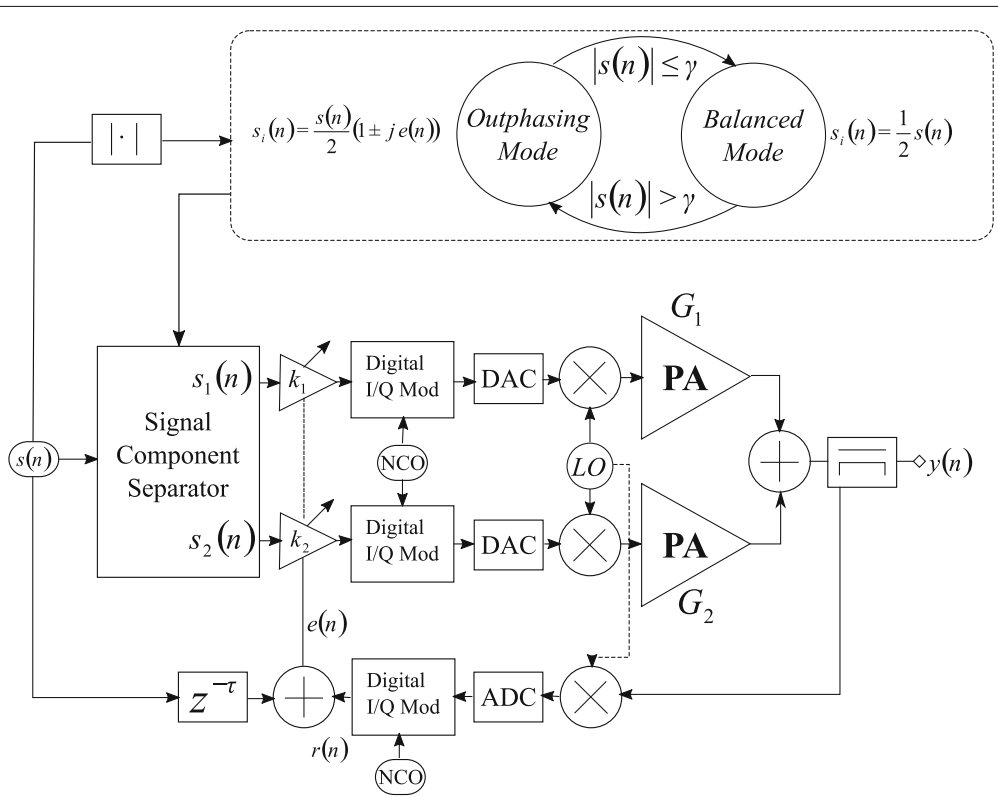

Fig. 1 General mode-multiplexing LINC architecture. The digital domain is carried out in a FPGA test-bench, whereas the remaining parts are analog components 
where the $\Lambda$ matrix is driven through a Markov chain and is defined as

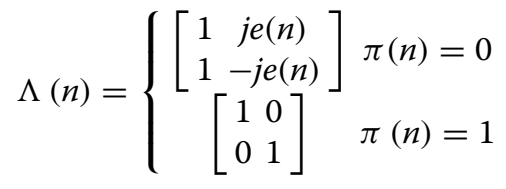

where $\pi(n)$ is the Markov state. The sequence of binaryvalued random samples $\pi(0), \pi(1), \ldots \pi(n)$ can be easily generated in a digital platform (DSP or FPGA) or can be offline created and stored in a RAM or ROM if desired to reduce the computational cost [12]. In addition, it should fulfill the so-called Markov property [13], and each binary value, i.e., the state value, controls the transmitting mode (“OUTPHASING (O)", "BALANCED (B)").

Figure 2 shows the architecture corresponding to the novel policy in comparison to the MM-LINC. The signal component separator for both schemes carries out the decomposition

$$
s_{1,2}=(1-\alpha(n)) \frac{s(n)}{2}(1 \pm j e(n))+\alpha(n) \frac{s(n)}{2}
$$

where $\alpha$ is a binary sequence which controls the statistical properties of the PA input signals, and consequently the trade-off between efficiency and linearity. The MMLINC sequence is based on the input samples (" 1 ," if the instantaneous envelope is greater than the threshold, "0" otherwise), and in our proposal, it is based on the mathematical properties of a Markov process.
The new switching policy is described by using the values of the conditional probabilities:

$$
\begin{aligned}
& P\left(\pi(n+1)={ }^{\prime} \mathrm{B}^{\prime} / \pi(n)={ }^{\prime} \mathrm{O}^{\prime}\right)=a \\
& P\left(\pi(n+1)={ }^{\prime} \mathrm{O}^{\prime} / \pi(n)={ }^{\prime} \mathrm{B}^{\prime}\right)=b
\end{aligned}
$$

where $a$ and $b$ are values specified by the designer. These are the probabilities of going from state BALANCED to OUTPHASING (or the opposite), in one time step. Besides, the sum of all the probabilities leaving a state must be one. Under this lossless chain assumption, the random state is modeled with the aid of a transition probability matrix which is given by

$$
M=\left(\begin{array}{cc}
1-a & a \\
b & 1-b
\end{array}\right)
$$

Furthermore, we study two magnitudes so that the effects of $a$ and $b$ can be investigated and the operative principles can be properly understood:

(i) Percentage of outphasing or balanced samples: the greater the number of LINC-transmitted samples, the higher the PA efficiency and the lower the isolated combiner efficiency. Those are estimated by means of the steady state probabilities, which are computed using (9) and

$$
P(\pi=0)+P(\pi=1)=P_{O}+P_{B}=1
$$

where $P_{O}$ is the probability of a sample of being transmitted in outphasing mode and $P_{B}$ of the balanced mode.

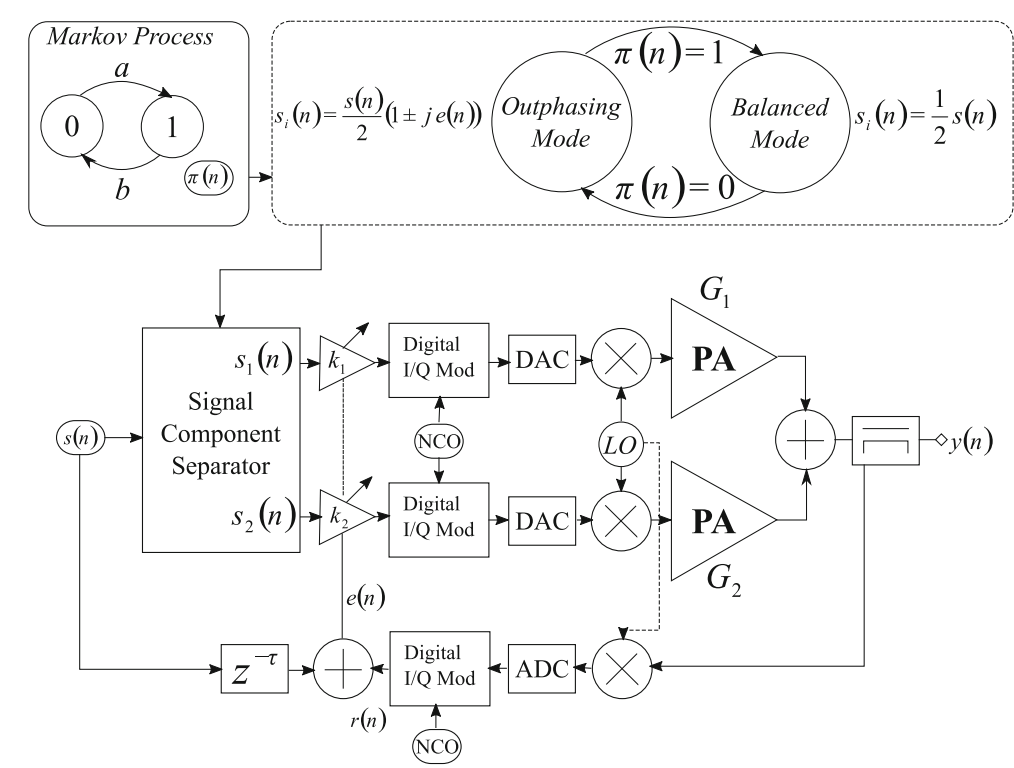

Fig. 2 Proposed Markov-multiplexed LINC architecture. The switching is controlled by a sequence which can be real-time generated or stored in a ROM using a state diagram 
a

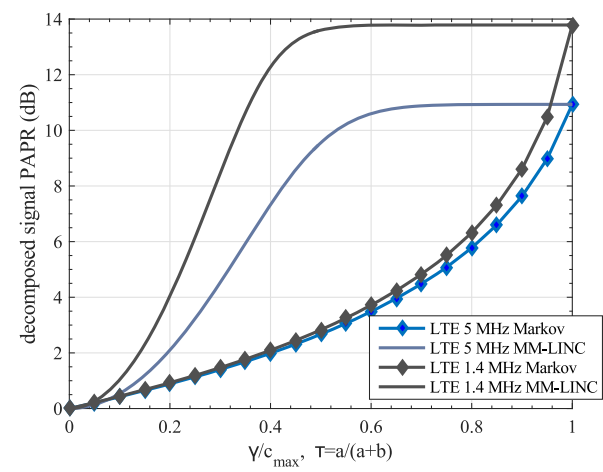

b

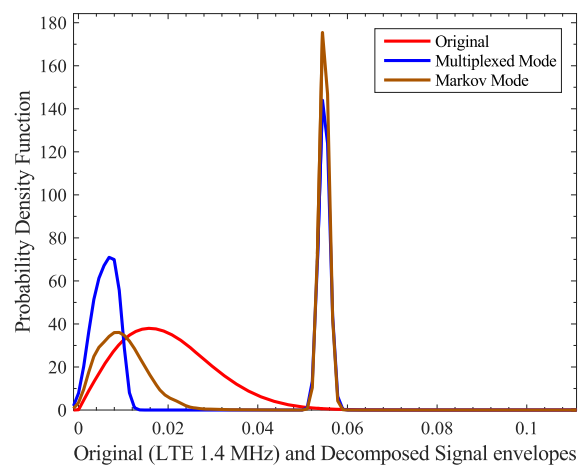

Fig. 3 PAPR and statistics parameters. a Input signal PAPR in terms of the policy parameters from closed-form expressions. $\mathbf{b}$ Example of pdfs for specific policy parameters

According to Markov theory,

$$
\left[\begin{array}{ll}
P_{O} & P_{B}
\end{array}\right]=\left[\begin{array}{ll}
P_{O} & P_{B}
\end{array}\right]\left(\begin{array}{cc}
1-a & a \\
b & 1-b
\end{array}\right)
$$

Solving (10) with (11),

$$
P_{B}=\frac{a}{a+b} \quad P_{O}=\frac{b}{a+b}
$$

(ii) The averaged time of being in outphasing (balanced) mode before changing to balanced (outphasing) mode: This property is related to how many samples are consecutively transmitted in the same mode (neglecting instantaneous envelope values). It quantifies the correlation properties of the signal and the switching rate between modes. According to Markov chain theory, the number of average samples for each state $\left(N_{O}, N_{B}\right)$ is computed as

$$
\begin{aligned}
& N_{O}=\frac{1}{P\left(\pi(n+1)=^{\prime} B^{\prime} / \pi(n)={ }^{\prime} O^{\prime}\right)}=\frac{1}{a} \\
& N_{B}=\frac{1}{P\left(\pi(n+1)=^{\prime} O^{\prime} / \pi(n)=^{\prime} B^{\prime}\right)}=\frac{1}{b}
\end{aligned}
$$

The importance of these equations will be addressed with the simulations and with analysis of the theoretical PAPR. Theory and results will be focused on OFDM-like modulations due to the widespread use in actual wireless communication systems, but the technique is applicable to other modulation formats such as WCDMA. Markov LINC reduces the PAPR of the decomposed signal in comparison to the mode multiplexing method, and consequently, there will be a noticeable difference between both with high PAPR waveforms.

\subsection{PAPR theorical performance}

In order to show that the proposed algorithm outperforms the existing multiplexing methods in OFDM, we present a novel theoretical analysis of the PAPR not only for the
Markov multiplexed architecture but also for the MMLINC. The PAPR of the transmitted signal, $s_{n}$ is defined as $[14,15]$

$$
\mathrm{PAPR}=10 \log \frac{\max \left|s_{n}\right|^{2}}{E\left[\left|s_{n}\right|^{2}\right]} d B
$$

where $E\left[\left|s_{n}\right|^{2}\right]$ is its averaged power. As far as a generic OFDM signal is concerned, it is assumed that the asymptotic probability distribution (pdf) follows a Rayleigh statistics since the inphase and quadrature components approximate to Gaussian processes,

$$
f_{|s|}(\rho)=\frac{2}{R} \rho e^{-\rho^{2} / R} \quad R=2 \sigma^{2} \quad \rho>0
$$

being $\sigma$ the inphase or quadrature variance.

On the other hand, LINC waveform envelopes are constant regardless the input signal values. Thus, it is easy to write the envelope statistics taking into account that LINC signals have an envelope amplitude of value $c_{\max } / 2$ as

$$
f_{\left|s_{n}\right|}(\rho)=\delta\left(\rho-c_{\max } / 2\right)
$$

being $\delta$ the Dirac distribution. Notice that in the case of the classical LINC, PAPR $=1$.

The terms in (14) can be evaluated in both multiplexing schemes by means of a proper envelope statistics closed-form expression. Let $\mathrm{F}$ be the probability function corresponding to the original input signal. Integrating (15), we obtain

$$
F(\alpha)=P(|s|<\alpha)=\int_{0}^{\alpha} f_{|s|}(\rho) d \rho
$$

Table 1 Input envelope statistical values and PAPR

\begin{tabular}{lllll}
\hline Bandwidth & $c_{\max }$ & $\mathrm{E}[\rho]$ & $\operatorname{Var}[\rho]$ & $\mathrm{PAPR}(\mathrm{dB})$ \\
\hline $1.4 \mathrm{MHz}$ & 0.1096 & 0.01974 & $1.19 \cdot 10^{-4}$ & 13.73 \\
$5 \mathrm{MHz}$ & 0.1654 & 0.04019 & $5.93 \cdot 10^{-4}$ & 10.93 \\
\hline
\end{tabular}


Table 2 Multiplexing-Mode LINC theoretical and real PAPR values

\begin{tabular}{llll}
\hline Bandwidth & Policy & Real PAPR (dB) & Pred. PAPR (dB) \\
\hline $1.4 \mathrm{MHz}$ & Reverse MM & 3.3487 & 3.2575 \\
& Markov & 2.9479 & 2.83018 \\
$5 \mathrm{MHz}$ & Reverse MM & 3.369 & 3.3111 \\
& Markov & 2.95 & 2.6734
\end{tabular}

$$
F_{\text {OFDM }}(\alpha)=1-e^{-\alpha^{2} / R} .
$$

\subsubsection{MM-LINC PAPR}

In the MM-LINC, the modes are switched using the $\gamma$ threshold. Thus, the probability distribution is computed in the balanced mode by constraining the envelope distribution to $\gamma$, which leads to

$$
\begin{aligned}
F_{|s| / B}(\rho) & =F_{s \mid / B}(\rho|| s \mid<\gamma)= \\
& =\frac{P(|s|<\rho,|s|<\gamma)}{P(|s|<\gamma)}=\left\{\begin{array}{cc}
\frac{P(|s|<\rho)}{P(|s|<\gamma)} & \text { if } \rho<\gamma \\
1 & \rho>\gamma
\end{array}\right.
\end{aligned}
$$

Therefore, the probability density function should be

$$
f_{|S| / B}(\rho)=\frac{f_{S}(\rho)}{F_{S}(\gamma)} u(\gamma-\rho)
$$

being $u(x)$ the Heaviside function. Finally, it is important to realize that the envelope is transmitted in the balanced mode with a $3 \mathrm{~dB}$ backoff. Using (15), (18), and (20) and a change of variable, it is found that

$g_{|S| / B}(\rho)=f_{S \mid / B}(2 \rho) 2=\frac{8 \rho e^{-4 \rho^{2} / R}}{R\left(1-e^{-\gamma^{2} / R}\right)} u\left(\frac{\gamma}{2}-\rho\right)$

The MM-LINC pdf is obtained applying the total probability theorem with the conditional probability in the LINC mode in (16) $f_{|s|}(\rho)=g_{|s| / B}(\rho) P(|s| \leq \gamma)+f_{|s| / O}(\rho)(1-P(|s| \leq \gamma)$

Using the results from (16), (18), and (21),

$f_{|s|}(\rho)=\frac{8 \rho e^{-4 \rho^{2} / R}}{R} u\left(\frac{\gamma}{2}-\rho\right)+\delta\left(\rho-\frac{c_{\max }}{2}\right) e^{-\gamma^{2} / R}$

If some samples are transmitted in outphasing mode, which is reasonable if the threshold $\gamma$ is properly selected, the numerator in (14) comes from the LINC constant envelope property, which yields to

$$
\max (|s|)=\frac{c_{\max }}{2}
$$

The denominator in (14) is computed using (23), as summarized in (35) in the Appendix. Finally, the closed-form PAPR expression in a MM-LINC scheme is

$$
\mathrm{PAPR}=\frac{c_{\max }{ }^{2}}{R-e^{-\gamma^{2} / R}\left(R+\gamma^{2}\right)+c_{\max }{ }^{2} e^{-\gamma^{2} / R}}
$$

\subsubsection{Markov-multiplexed LINC PAPR}

The novel Markov scheme PAPR estimation is analogous to the MM-LINC mode with certain differences such as the pdf in the case of the balanced mode and the probabilities of each mode, which are now those in (12). In addition, the maximum envelope is limited in the balanced state to $c_{\max } / 2$ instead of $\gamma$, which is usually less than $c_{\max } / 2$. This stems from the fact that the switching policy is precisely independent from the signal amplitude and consequently

$$
g_{s \mid B}(\rho)=\frac{8 \rho e^{-4 \rho^{2} / R}}{R\left(1-e^{-c_{\max } / R}\right)} u\left(\frac{c_{\max }}{2}-\rho\right)
$$

The conditional probability corresponding to the outphasing mode still remains. Thus,
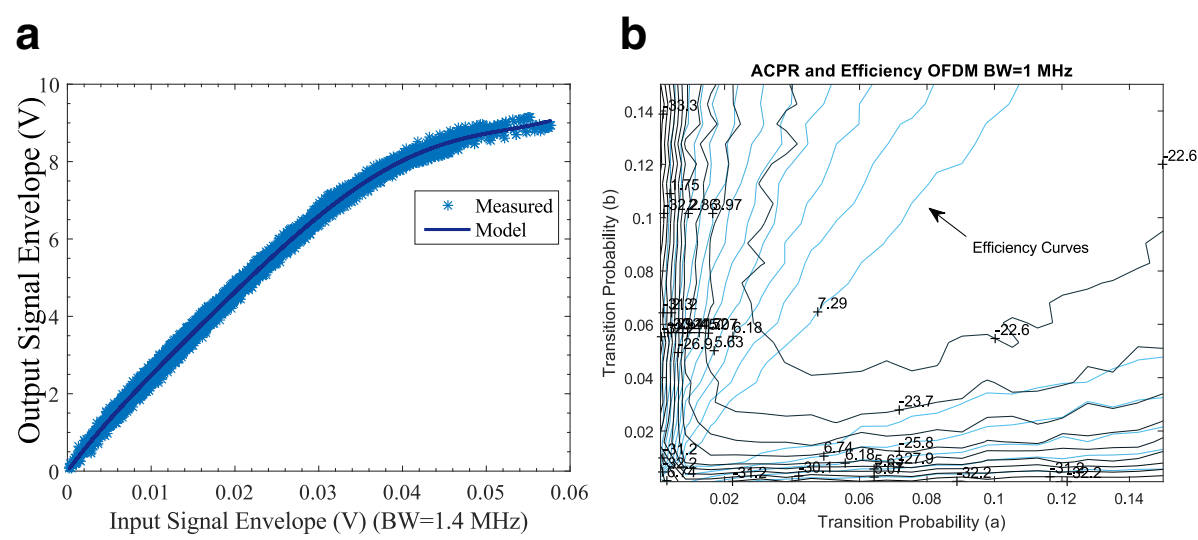

Fig. 4 Markov simulation elements. a AM-AM Cree PA-measured and PM-identified model. b $1.4 \mathrm{MHz}$ Markov-LINC ACPR performance in terms of transition probabilities 


\section{a}

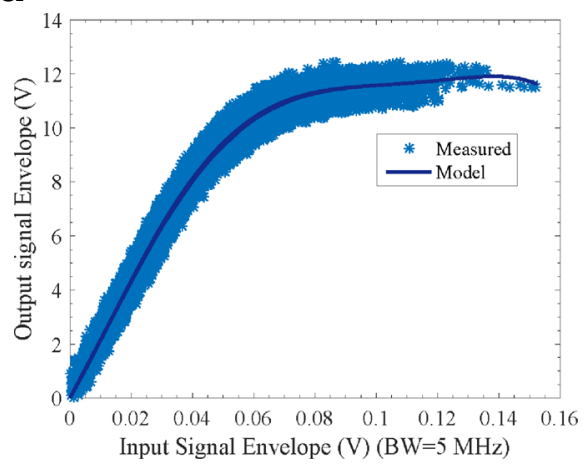

b

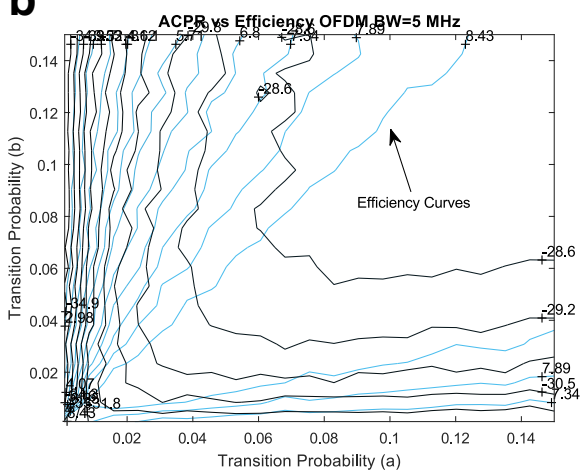

Fig. 5 Markov simulation elements. a AM-AM Cree PA-measured and PM-identified model. b 5 MHz Markov-LINC ACPR Performance in terms of transition probabilities

$$
f_{|s|}(\rho)=g_{|s| / B}(\rho) P_{B}+f_{|s| / O}(\rho) P_{0}
$$

Replacing (12), (16), and (26) in (27),

$$
\begin{aligned}
f_{|s|}(\rho)= & \frac{8 \rho e^{-4 \rho^{2} / R}}{R\left(1-e^{-c_{\max } / R}\right)} u\left(\frac{c_{\max }}{2}-\rho\right) \frac{a}{a+b} \\
& +\delta\left(\rho-\frac{c_{\max }}{2}\right) \frac{b}{a+b}
\end{aligned}
$$

The PAPR numerator is $c_{\max }^{2} / 4$ in the Markov mode, and the averaged power is estimated in the Appendix in (37). According to those results,

$$
\text { PAPR }=\frac{c_{\max }^{2}}{\left(R-\frac{c_{\max ^{2}}{ }^{2}}{e^{\max ^{2} / R}-1}\right) \frac{a}{a+b}+c_{\max } 2 \frac{b}{a+b}}
$$

The PAPR Eqs. (25) and (29) may forecast suitable values for $\gamma$ and the relation $a / b$ in order to fix a specified PAPR after estimating the input original OFDM signal statistical moments. Two practical waveforms test the proposed equation validity in this work, a QPSK OFDM downlink signal with 1.4 and $5 \mathrm{MHz}$ bandwidth. The decomposition process is accomplished by means of the Matlab platform.

Equations (25) and (29) are studied under threshold variations, $(\gamma)$, and the steady-state probability $\tau=P_{B}$. According to Fig. 3a, both methods can achieve PAPR values between $0 \mathrm{~dB}$ (fully LINC mode) and the PAPR of the original signal. However, the Markov decomposed signal PAPR is lower for a wider range of $a$ and $b$ values and increases quickly when $\tau$ approaches the complete balanced state. As expected, both policies agree in the limiting cases (LINC and balanced limits).

Table 1 shows their statistical theoretical and estimated values with selected fixed parameters $(\gamma=E[(|s(n)|]$ and $a=b=0.01$ ). The prediction (Table 2) agrees with those numerically calculated, but there is a small difference in the Markov case. This is due to fact that the estimated value is a single realization of the process, whereas (29) computes averaged values.

Figure $3 \mathrm{~b}$ shows the probability density functions not only of the OFDM signal envelopes, which are well approximated using the Rayleigh distribution, but also of
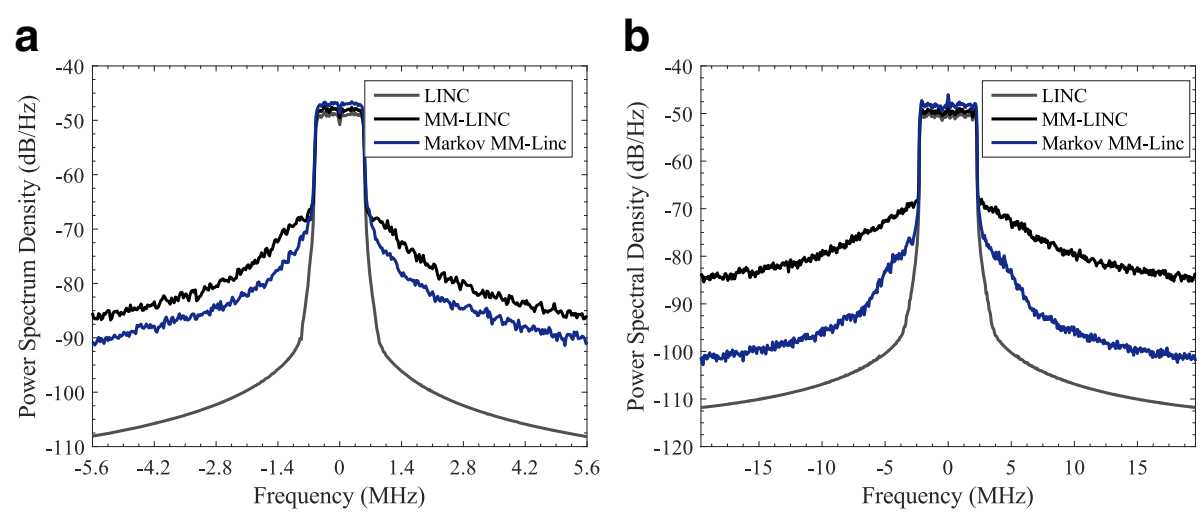

Fig. 6 Simulation results with LINC, MM-LINC, Markov-LINC, and direct amplification. a OFDM with $1.4 \mathrm{MHz}$ bandwidth. b OFDM with $5 \mathrm{MHz}$ bandwidth 
Table 3 Estimated efficiency bounds (\%) OFDM $1 \mathrm{MHz}$ bandwidth

\begin{tabular}{llllll}
\hline Conf. & Class A & Class B & Comb. & Total A & Total B \\
\hline LINC & 25 & 39.27 & 4.23 & 1.06 & 1.66 \\
MMLINC & 12.87 & 20.21 & 57.98 & 7.45 & 11.72 \\
Markov & 14.36 & 22.56 & 53.84 & 7.73 & 12.15 \\
\hline
\end{tabular}

the classical multiplexing mode and the Markov mode envelopes. Their pdfs have strong peaks in the $c_{\max } / 2$ envelope value, which are clearly outphased LINC samples. According to Fig. 3b, there are strong differences in the balance mode statistics which leads to different performance in terms of efficiency and linearity.

\section{Simulation Analysis}

The importance of the Markov chain parameters $a$ and $b$ has been shown in previous sections. On the one hand, PAPR can be selected according to the percentage of outphased signal samples. On the other hand, this allows us to properly set the ratio $a / b$ but not to fix numerical values for $a$ and $b$. Distortion analysis can be helpful in order to finally choose suitable values which aim at obtaining good performance in terms of linearity and efficiency. Markov LINC simulations are carried out to study the performance and to compare to the MM-LINC and classical LINC. Firstly, a PA model is extracted from real measurements using a polynomial model (PM) with an OFDM signal source. Secondly, an ACPR analysis is addressed using the PA models to draw some conclusions about $a$ and $b$ independently. Finally, the LINC structure is evaluated in an ideal scenario (both branches do not have imbalances).

Figures $4 \mathrm{a}$ and $5 \mathrm{a}$ show the measurement and the simple model corresponding to a real power amplifier. Polynomial models are a very popular class of behavioral power amplifier mathematical representations for wideband applications and or high-power amplifiers and are shown to be a good characterization of nonlinear PAs [16-18]. If the baseband input and output are $x(t), y(t)$ respectively, $\phi_{p}$ is a basis function with terms in the form of $|x(t)|^{p} x(t), \alpha_{p}$ are complex coefficients, $N$ is the nonlineary order, the PM:

$$
y(n)=\sum_{p=0}^{N} \alpha_{p} \phi_{p}(n)=x(n) \sum_{p=0}^{N} \alpha_{p}|x(n)|^{p}
$$

In this work, the real modeled PA is the CREE CGH40006P, which has 1-dB compression around $29.9 \mathrm{dBm}$ and is measured around $2.6 \mathrm{GHz}$ using multicarrier modulation with two different signal bandwidths, 1.4 and $5 \mathrm{MHz}$. Finally, the PM model has been trained up to order 7 .
Transition probabilities are the key parameters which may be analyzed to minimize spectral regrowth. Some Monte Carlo simulations have been carried out to study their effects on ACPR and efficiency. Figures $4 \mathrm{~b}$ and $5 \mathrm{~b}$ show that better efficiencies are achieved when the transition probabilities $a$ and $b$ are relatively close. Intuitively, if combiner and PA efficiencies would increase/decrease at the same rate, then a $50 \%$ would be the optimal value in terms of efficiency and distortion. The efficiency rate depends on the bias of the PAs and is different from that of the combiner. Thus, the trade-off solution is not exactly at this point but close. As a conclusion, some optimization algorithm should be carried to choose the accurate proportion between outphasing and balanced samples in order to fulfill a standard specification.

In addition, the higher values of transition probabilities, the faster the modes switch $\left(N_{B}, N_{O}\right.$, are smaller) due to the time variability of the signals. The Markov sequence multiplies the samples, and $a$ and $b$ must fulfill the sampling theorem. Therefore, the signal bandwidth plays an important role so that the transition probabilities do not cause aliasing in the signal component separation process.

Figure 6 shows the simulation results obtained for a LINC structure in three cases, LINC, MM-LINC, and Markov LINC. The signal sources are the previous OFDM signals and are decomposed using the three schemes keeping the same input power. The MM-LINC threshold and transition probabilities are set using the PAPR and ACPR studies $(\gamma=0.18, a=b=0.01)$ just to check if the proposed technique works. These values have been chosen so that the output power with both multiplexing schemes were similar in order to have an efficiency which is comparable. Alternatively, those values could have been chosen to provide similar ACPR and then verify if the Markov technique provides better efficiency. Nevertheless, a proper optimization algorithm should be investigated or a utility function should be defined in order to choose the best trade-off according to the needs and requirements of a specific wireless scenario.

As expected, the Markov MM-LINC outperforms the standard mode multiplexing method because its signals have a better PAPR, leading to identical output power but less adjacent channel interference.

Table 4 Estimated efficiency bounds (\%) OFDM $5 \mathrm{MHz}$ bandwidth

\begin{tabular}{llllll}
\hline Conf & Class A & Class B & Comb. & Total A & Total B \\
\hline LINC & 25 & 39.27 & 8.07 & 2.02 & 3.17 \\
MMLINC & 13.07 & 20.53 & 62.03 & 8.11 & 12.73 \\
Markov & 16.55 & 26 & 49.75 & 8.23 & 12.93 \\
\hline
\end{tabular}



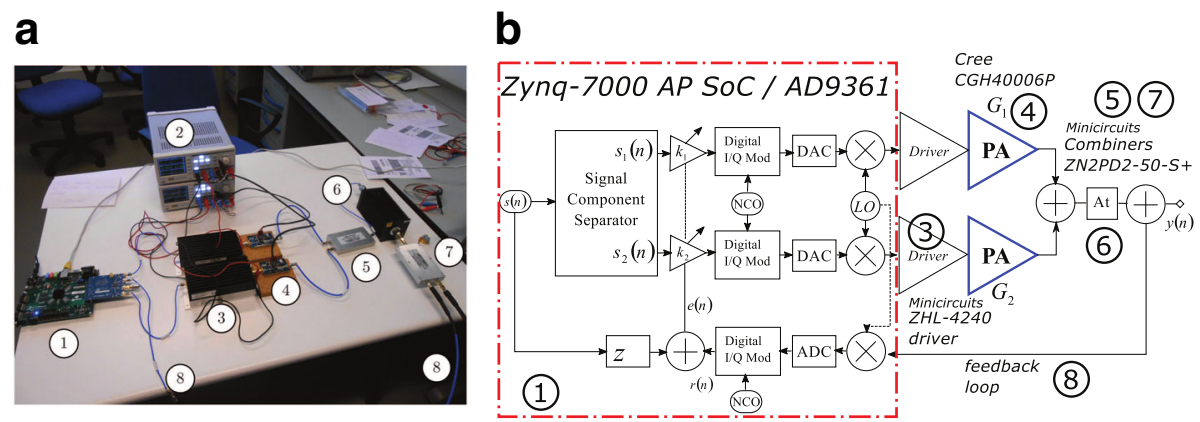

Fig. 7 Experimental setup. a Hardware implementation. b Equivalent black-box diagram corresponding to experimental test

Furthermore, efficiency bounds (showed in Tables 3 and 4) have been estimated in both cases, using results from $[10,11,14]$ under the hypothesis that the amplifier could be either class A biased or class B and with an isolated combiner. Actual values will be experimentally evaluated, but this shows that with the proposed values, the Markov method may lead to comparable efficiency values with a better ACPR behavior. The use of class B amplifiers could improve the efficiency but mode multiplexing schemes would be more sensitive to nonlinear effects.

\section{Experimental evaluation}

\subsection{Implementation}

Three mode-multiplexing algorithms have been tested on a LINC prototype to verify the real improvement and to find out if the trends predicted through simulations are accurate.

The complete experimental test bench is shown in Fig. 7a together with an equivalent block diagram including the real RF elements (Fig. 7b). The digital development platform used for the implementation of signal component separator, digital signal processes, and the digital I/Q modulator and demodulator consists of a main board (ZedBoard featuring Zynq 7020 All-Programmable SoC) connected to a PC, which controls a high-speed analog module with an integrated $\mathrm{RF}$ agile transceiver, the AD-FMCOMMS2-EBZ. It comprises a RF $2 \times 2$ transceiver with integrated 12-bit DACs and ADCs and has tunable channel bandwidth (from $200 \mathrm{kHz}$ to $56 \mathrm{MHz}$ ) and RX gain control. The output signals of the DACs are preamplified and upconverted to a RF frequency of 2 . $6 \mathrm{GHz}$. Finally, two power stages (a ZHL-4240 Minicircuits driver and a CGH400006P power amplifier) amplify both signals. The operating input power levels ensure that the driver is working linearly in back-off with the tested OFDM signals. Both branch outputs are combined by means of a power combiner (ZN2PD2-50-S+). The experimental evaluation begins with a calibration process, estimating the imbalance in magnitude and phase for each branch of the LINC transmitter. The imbalance correction algorithm uses the feedback loop and the RX AD-FMCOMMS2-EBZ input, and only two complex

\section{a}

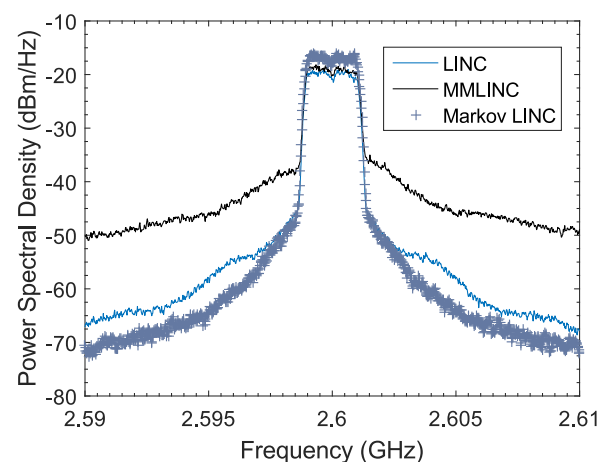

b

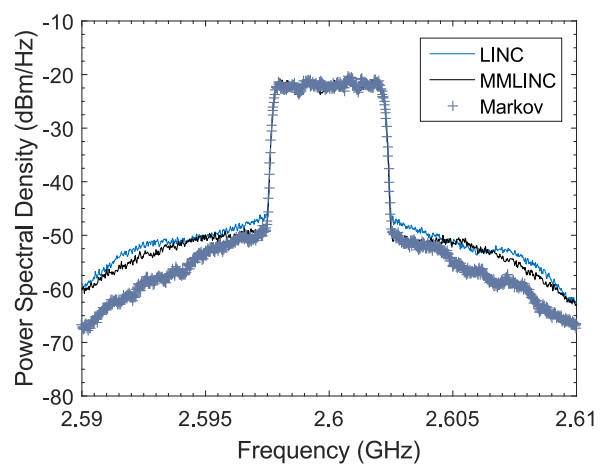

Fig. 8 Experimental performance results. Measured power spectrum densities in LINC, MM-LINC, and proposed approach (red). a OFDM 1.4 MHz and $\mathbf{b}$ OFDM $5 \mathrm{MHz}$ 
Table 5 Experimental LINC and mode-multiplexing results BW = $1.4 \mathrm{MHz}$

\begin{tabular}{llllllll}
\hline BW $=1.4 \mathrm{MHZ}$ & Pout $(\mathrm{dBm})$ & ACPR $(\mathrm{dBC})$ & $P_{(\mathrm{DC}, 1)}$ & $P_{(\mathrm{DC}, 2)}$ & Eff \% & FoM & EVM \% \\
\hline LINC & 26.3 & 29.3 & 13.32 & 12.35 & 1.66 & 1 & 2.47 \\
MM-LINC & 27.1 & 18.9 & 7.84 & 7.5 & 3.34 & 2.01 & 4.1 \\
Markov & 29.1 & 30.3 & 10.33 & 9.07 & 4.16 & 2.51 & 8.7 \\
\hline
\end{tabular}

coefficients (thus, it is only accurate in a narrowband context and not applicable to wideband waveforms). The transmitted signals, $\hat{s}_{1}(n)$ and $\hat{s}_{2}(n)$ are a corrected version in amplitude $k_{i}$ and phase $\phi_{i}$ of the outphasing signals $s_{1}(n)$ and $s_{2}(n)$ through two complex coefficients

$$
\begin{aligned}
& \hat{s}_{1}(n)=k_{1} e^{j \phi_{1}} s_{1}(n) \\
& \hat{s}_{2}(n)=k_{2} e^{j \phi_{2}} s_{2}(n)
\end{aligned}
$$

Those coefficients are computed by minimizing the ACPR using the Nelder-Mead algorithm which avoids to compute derivatives and can be adaptively performed. Therefore, the ACPR is a function $f$ of the correction coefficients,

$$
\operatorname{ACPR}(y)=f\left(k_{1}, \phi_{1}, k_{2}, \phi_{2}\right)
$$

and the optimal solution is

$$
\left(k_{1}^{o p t}, \phi_{1}{ }^{o p t}, k_{2}^{o p t}, \phi_{2}^{o p t}\right)=\arg \min \text { ACPR }\{y\}
$$

where $y(n)$ is an attenuated version at the combiner output. Once they are compensated, the signals corresponding to the different signal separation component methods are transmitted.

It must be pointed out that simulations do not include either memory effects or imbalances in the whole RF chain; hence, simulated and experimental figures are not directly comparable. Simulations are used in this case for designing the algorithms and the real setup is used for confirming the proposed approach.

In order to ensure that linearity is achieved without decreasing efficiency, we propose to use a figure of merit based on DC power $\left(P_{\mathrm{DC}}\right)$ and output power $\left(P_{\text {out }}\right)$, which are measured using the power supply and a spectrum analyzer (EXA N9010A). The total LINC efficiency (PA and combiner) can be evaluated as

$$
\eta_{\mathrm{LINC}}=\frac{P_{\text {out }}}{P_{\mathrm{DC}, 1}+P_{\mathrm{DC}, 2}}
$$

where subscripts 1 and 2 denote the DC power used by each power amplifier. This expression is also valid in the case of the mode-multiplexed policies. Therefore, the metric

$$
\text { FoM }=\frac{\eta_{\text {Markov }}}{\eta_{\text {LINC }}}=\frac{P_{\text {out }}^{\text {Markov }}}{P_{\text {out }}^{\mathrm{LINC}}} \cdot \frac{P_{\mathrm{DC}, 1}^{\mathrm{LINC}}+P_{\mathrm{DC}, 2}^{\mathrm{LINC}}}{P_{\mathrm{DC}, 1}^{\mathrm{Markov}}+P_{\mathrm{DC}, 2}^{\mathrm{Markov}}}
$$

takes into account the improvement or degradation in efficiency.

\subsection{Results}

The experimental setup has been tested with the same simulated OFDM signals and a sampling frequency of 61.44 MHz. Figure 8 shows the power spectral density of the output transmitter signal in a standard LINC, a MMLINC, and our proposed method. From a qualitative point of view, the standard LINC achieves good ACPR with the lowest output power as Tables 5 and 6 show. Better efficiency is obtained with a MM-LINC policy, because the output power is increased and DC power is decreased. However, the enhancement in output power is achieved at the same time that nonlinearity effects are increased. ACPR is increased compared to the standard LINC. The novel approach improves global efficiency as well and may provide the maximum output power compared to the other methods $(2 \mathrm{~dB}$ for the $1.4 \mathrm{MHz}$ signals and $0.9 \mathrm{~dB}$ for $5 \mathrm{MHz}$ ), with a higher architecture efficiency than the standard LINC. However, like in the MM-LINC policy, the EVM is increased in comparison to the standard LINC. Furthermore, the ACPR is the same than the standard LINC or even is improved $2.9 \mathrm{~dB}$, and the spectral regrowth is less severe in the Markov policy if it is compared to the MM-LINC scheme.

Although impairments are not studied in simulations, experimental results show their effects on the $5 \mathrm{MHz}$ OFDM signal. It should require a more robust memory mismatch correction algorithm to improve out-of-band cancellation, and in contrast, Markov MM-LINC waveforms seem to be less sensitive to RF chain imbalances, as the ACPR is better than in the standard LINC. The Markov-outphased signals do not have such a great signal bandwidth compared to the LINC signals, and therefore, impairments due to memory effects are small in comparison with the standard LINC and easier out-of band cancellation can be carried out. To sum up, the proposed method can comparatively achieve similar results than the MM-LINC method but with better ACPR, which is one of the characteristics of the reverse MM-LINC.

\section{Conclusion}

In this work, a novel mode multiplexing method for LINC transmitters is presented, implemented, and verified. A mathematical analysis of the PAPR proves that the new policy behaves better in terms of spectral

Table 6 Experimental LINC and mode-multiplexing results BW = $5 \mathrm{MHz}$

\begin{tabular}{llllllll}
\hline BW $=5 \mathrm{MHz}$ & Pout $(\mathrm{dBm})$ & ACPR $(\mathrm{dBC})$ & $P_{(D C, 1)}$ & $P_{(D C, 2)}$ & Eff \% & FoM & EVM \% \\
\hline LINC & 28.1 & 31.9 & 11.12 & 11.48 & 2.86 & 1 & 7.05 \\
MM-LINC & 28.3 & 31.4 & 6.44 & 6.92 & 5.06 & 1.77 & 9.28 \\
Markov & 29.2 & 34.8 & 7.84 & 7.7 & 7.7 & 1.87 & 11.16
\end{tabular}


regrowth compared to other proposed multiplexing policies, which has been verified by means of simulation. The experimental LINC transmitter has been implemented and tested with OFDM signals, obtaining good performance with low complexity. Experimental results demonstrate that it is possible to reduce the ACPR up to $4.5 \mathrm{~dB}$ in a system with a multicarrier modulation without decreasing the output mean power, compared to standard MM methods, and improving efficiency by approximately 2 compared to the classical LINC technique.

\section{Appendix}

According to (14), PAPR derivation requires to compute the quantity

$$
P_{a v}=E\left[\left|s_{n}\right|^{2}\right]=\int \rho^{2} f_{|s|}(\rho) d \rho
$$

The MM-LINC PAPR uses the density given in (23). Then

$$
\begin{aligned}
& P_{a \nu}^{\mathrm{MM}-\mathrm{LINC}}=\int \rho^{2} f_{|s|}^{\mathrm{MM}-\operatorname{LINC}}(\rho) d \rho= \\
& =\int_{0}^{\gamma / 2} \frac{8}{R} \rho^{3} e^{-4 \rho^{2} / R} d \rho \\
& +\int_{0}^{\infty} \rho^{2} e^{-\gamma^{2} / R} \delta\left(\rho-\frac{c_{\max }}{2}\right) d \rho \\
& =-\left.\frac{1}{4} e^{-4 \rho^{2} / R}\left(R+4 \rho^{2}\right)\right|_{0} ^{\gamma / 2}+\left(\frac{c_{\max }}{2}\right)^{2} e^{-\gamma^{2} / R}
\end{aligned}
$$

Simplifying

$P_{a \nu}^{\mathrm{MM}-\mathrm{LINC}}=\frac{1}{4}\left[R-e^{-\gamma^{2} / R}\left(R+\gamma^{2}\right)\right]+\left(\frac{c_{\max }}{2}\right)^{2} e^{-\gamma^{2} / R}$

The Markov-multiplexed LINC PAPR uses the density given in (28). Then

$$
\begin{aligned}
P_{a v}^{\text {Markov }}= & \int \rho^{2} f_{|s|} \text { Markov }(\rho) d \rho= \\
= & \int_{0}^{c_{\max } / 2} \frac{8 \rho^{3} e^{-4 \rho^{2} / R}}{R\left(1-e^{-c_{\max } / R}\right)} \frac{a}{a+b} d \rho \\
& +\int_{0}^{\infty} \rho^{2} \delta\left(\rho-\frac{c_{\max }}{2}\right) \frac{b}{a+b} d \rho
\end{aligned}
$$

Computing the integrals,

$P_{a \nu}^{\text {Markov }}=\frac{1}{4}\left(R-\frac{c_{\max }^{2}}{e^{c_{\max }^{2} / R}-1}\right) \frac{a}{a+b}+\left(\frac{c_{\max }}{2}\right)^{2} \frac{b}{a+b}$

\section{Abbreviations}

ACPR: Adjacent channel power ratio; ADC: Analog digital converter; DAC: Digital analog converter; DSP: Digital signal processor; EER: Envelope elimination and restoration; FBMC: Filtered band multicarrier; FPGA: Field programmable gate array; GFDM: Generalized frequency division multiplexing; LINC: Linear amplification with non-linear components; LTE: Long-term evolution; MILC: Modified implementation of LINC; MM-LINC: Modemultiplexing LINC; OFDM: Orthogonal frequency multiplexing modulation; PA: Power amplifier; PAPR: Peak-to-average power ratio; PC : Personal computer; PDF: Probability density function; QPSK: Quadrature phase-shift keying; RAM: Random access memory; RF: Randiofrequency; RM-LINC: Reverse mode LINC; ROM: Read-only memory; SCS: Signal component separator

\section{Funding}

This work was supported by the spanish Government (Project TEC2014-58341-C4-2-R from MICINN and FEDER) and the Aragón Government and FSE (GCM T97).

\section{Authors' contributions}

PLC contributed to the measurements, result analysis, and article writing. PG-D contributed to the result analysis and article writing. JdeM contributed to the result analysis and article writing. AV contributed to the article writing. All authors read and approved the final manuscript.

\section{Competing interests}

The authors declare that they have no competing interests.

\section{Publisher's Note}

Springer Nature remains neutral with regard to jurisdictional claims in published maps and institutional affiliations.

Received: 31 May 2017 Accepted: 22 January 2018

Published online: 12 February 2018

\section{References}

1. PM Lavrador, TR Cunha, PM Cabral, JC Pedro, The linearity efficiency compromise. IEEE Microw. Mag. 11(5), 44-58 (2010)

2. A Birafane, M El-Asmar, AB Kouki, M Helaoui, FM Ghannouchy, Analyzing LINC systems. IEEE Microw. Mag. 11(5), 59-71 (2010)

3. B Kim, J Moon, I Kim, Efficiently Amplified. IEEE Microw. Mag. 11(5), 87-99 (2010)

4. M Helaoui, FM Ghannouchi, Linearization of power amplifiers using the reverse MM-LINC technique. IEEE Trans. Circuits Syst. II, Exp. Briefs. 57(1), 6-10 (2010)

5. M Helaoui, S Boumaiza, FM Ghannouchi, AB Kouki, A Ghazel, A new modemultiplexing LINC architecture to boost the efficiency of WiMAX up-link transmitters. IEEE Trans. Microw. Theory Techniques. 55(2), 248-253 (2007)

6. P Garcia-Ducar, A Ortega, J de Mingo, A Valdovinos, Nonlinear distortion cancellation using LINC transmitters in OFDM system. IEEE Trans. Broadcast. 51(1), 84-93 (2005)

7. P Garcia-Ducar, J de Mingo, PL Carro, A Valdovinos, Design and experimental evaluation of a LINC transmitter for OFDM systems. IEEE Trans. Wirel. Commun. 9(10), 2983-2987 (2010)

8. THwanq, K Azadet, RS Wilson, J Lin, Linearization and imbalance correction techniques for broadband outphasing power amplifiers. IEEE Trans. Microw. Theory Tech. 63(7), 248-253 (2015)

9. AF Aref, TM Hone, R Negra, A study of the impact of delay mismatch on linearity of outphasing transmitters. IEEE Trans. Circ. Syst. I. 62(1), 254-262 (2015)

10. FH Raab, Efficiency of outphasing RF power-amplifier systems. IEEE Trans. Commun. 33(10), 1094-1099 (1985)

11. T Hwanq, K Azadet, RS Wilson, J Lin, On the linearity and efficiency of outphasing microwave amplifiers. IEEE Trans. Microw. Theory Tech. 52(7), 1702-1708 (2004)

12. PL Gilabert, A Cesari, G Montoro, E Bertrand, J Dilhac, Multi-lookup table FPGA implementation of an adaptive digital predistorter for linearizing RF power amplifiers with memory effects. IEEE Trans. Microw. Theory Tech. 56(2), 372-384 (2008)

13. H Yang, M Alouini, Markov chains and performance comparison of switched diversity systems. IEEE Trans. Commun. 52(7), 1113-1125 (2004)

14. $\mathrm{H}$ Ochiai, $\mathrm{H}$ imai, On the distribution of the peak-to-average power ratio in OFDM signals. IEEE Trans. Commun. 49(2), 282-289 (2001)

15. H Ochiai, An analysis of band-limited communication systems from amplifier efficiency and distortion perspective. IEEE Trans. Commun. 61(4), 282-289 (2013) 
16. DR Morgan, Z Ma, J Kim, MG Zierdt, J Pastalan, A generalized memory polynomial model for digital predistortion of RF power amplifiers. IEEE Trans. Microw. Theory Tech. 54(10), 3852-3860 (2006)

17. K Hyunchul, JS Kenney, Behavioral modeling of nonlinear RF power amplifiers considering memory effects. IEEE Trans. Microw. Theory Tech. 51(12), 2495-2504 (2003)

18. W Huadong, B Jingfu, W Zhengdu, Comparison of the behavioral modelings for RF power amplifier with memory effects. IEEE Microw. Wireless Components Lett. 19(3), 179-181 (2009)

\section{Submit your manuscript to a SpringerOpen ${ }^{\circ}$ journal and benefit from:}

- Convenient online submission

- Rigorous peer review

- Open access: articles freely available online

- High visibility within the field

- Retaining the copyright to your article

Submit your next manuscript at $\gg$ springeropen.com 